\title{
Fragmentation of Neutral Amino Acids and Small Peptides by Intense, Femtosecond Laser Pulses
}

Article in Journal of the American Society for Mass Spectrometry · July 2013

DOI: 10.1007/s13361-013-0653-6 · Source: PubMed

CITATIONS

15 authors, including:

Martin J Duffy

Queen's University Belfast

24 PUBLICATIONS 387 CITATIONS

SEE PROFILE

John Costello

Dublin City University

203 PUBLICATIONS $\mathbf{3 , 8 5 9}$ CITATIONS

SEE PROFILE
134

Raymond King

Queen's University Belfast

47 PUBLICATIONS 219 CITATIONS

SEE PROFILE

David J Timson

University of Brighton

197 PUBLICATIONS $\quad 2,795$ CITATIONS

SEE PROFILE

Some of the authors of this publication are also working on these related projects:

Simple derivatisation of the old coccidiostat decoquinate to provide new 'drug-like' derivatives suitable for treatment of malaria and other parasitic diseases, and TB View project

Tang Ben Zhong View project 


\title{
Fragmentation of Neutral Amino Acids and Small Peptides by Intense, Femtosecond Laser Pulses
}

\author{
M.J. Duffy, ${ }^{a}$ O. Kelly, ${ }^{a}$ C.R. Calvert, ${ }^{a}$ R.B. King, ${ }^{a}$ L. Belshaw, ${ }^{a}$ T. J. Kelly, ${ }^{b}$ J. T. Costello, ${ }^{b}$ D. J. \\ Timson, ${ }^{\mathrm{c}}$ W.A. Bryan, ${ }^{\mathrm{d}}$ T. Kierspel, ${ }^{\mathrm{e}}$ I.C.E. Turcu, ${ }^{\mathrm{e}}$ C.M. Cacho, ${ }^{\mathrm{e}}$ E. Springate, ${ }^{\mathrm{e}}$ I.D. Williams, ${ }^{\mathrm{a}}$ and \\ J.B. Greenwood ${ }^{\mathrm{a}}$
}
${ }^{a}$ Centre for Plasma Physics, School of Mathematics and Physics, Queen's University Belfast, BT7 $1 \mathrm{NN}, \mathrm{UK}$.
${ }^{\mathrm{b}}$ National Centre for Plasma Science and Technology, School of Physical Sciences, Dublin City University, Republic of Ireland

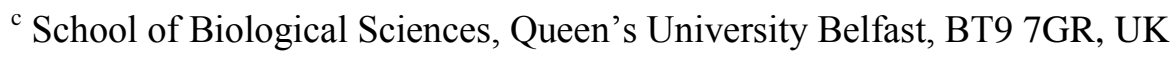

${ }^{\mathrm{d}}$ Department of Physics, Swansea University, Swansea, SA2 8PP, UK

${ }^{\mathrm{e}}$ STFC Rutherford Appleton Laboratory, Didcot, Oxon, OX11 0QX, UK

\begin{abstract}
High power femtosecond laser pulses have unique properties which could lead to their application as ionization or activation sources in mass spectrometry. By concentrating many photons into pulse lengths approaching the timescales associated with atomic motion, very strong electric field strengths are generated which can efficiently ionize and fragment molecules without the need for resonant absorption. However, the complex interaction between these pulses and biomolecular species is not well understood. To address this issue, we have studied the interaction of intense, femtosecond pulses with a number of amino acids and small peptides. Unlike previous studies, we have used neutral forms of these molecular targets which allowed us to investigate dissociation of radical cations
\end{abstract}


without the spectra being complicated by the action of mobile protons. We found fragmentation was dominated by fast, radical-initiated dissociation close to the charge site generated by the initial ionization or from subsequent ultrafast migration of this charge. Fragments with lower yields which are useful for structural determinations were also observed and attributed to radical migration caused by hydrogen atom transfer within the molecule.

Address reprint requests to Jason Greenwood, Centre for Plasma Physics, Queen's University Belfast, Belfast BT7 1NN, UK. Email: j.greenwood@qub.ac.uk 


\section{Introduction}

The identification and sequencing of biopolymers, especially proteins, is one of the most significant applications of mass spectrometry, where sequences of sub-units are determined by measuring mass differences from the fragment ions produced following activation of the molecule. However, the use of conventional activation techniques for proteomics rarely yields complete sequence information, making top-down, de novo sequencing difficult and limiting studies of post-translational modifications or other classes of molecule. Recently, alternative photo-activation methods employing wavelengths from the mid-infrared to vacuum ultraviolet have been investigated. While these new laser fragmentation techniques are at an early stage of exploitation, they are likely to play a prominent role in tandem mass spectrometry in the future [1]. A novel development in this area has been the use of high power femtosecond laser pulses for both ionization and fragmentation in mass spectrometers, where resonant excitation of the molecule is not necessarily required. The high photon density and strong fields of these pulses can induce multi-photon or tunnelling ionization with an efficiency which is orders of magnitude greater than conventional electron impact and can produce an extensive range of fragment ions which are ideal for structural analysis.

The outcome of the interaction of a femtosecond laser pulse with a molecule depends on laser characteristics such as wavelength, pulse length, intensity, and polarization as well as the nature of the molecular system being studied. For femtosecond lasers, pulse lengths are similar to, or shorter than, characteristic timescales for nuclear motion resulting in many dissociation pathways being bypassed due to a ladder climbing process to the continuum through real or virtual excited states. This produces a higher proportion of parent ions than is typically obtained by electron impact, which is highly desirable for chemical identification. As these ion sources are also pulsed, they are readily coupled to time of flight mass spectrometers or ion traps [2] and have already found practical applications. For instance it has been demonstrated that molecules can be very efficiently ionized at atmospheric pressure and injected into the ion guide of a mass spectrometer [3], and recently dioxin contamination was detected in samples acquired following the Tōhoku earthquake and resulting tsunami in 2011 [4]. 
As well as being able to detect chemicals at the femtogram level [5], the lower fragmentation reduces the need for pre-processing of the sample, thus simplifying and speeding up the analysis.

In addition to direct chemical identification which requires minimal fragmentation of the parent molecules, femtosecond pulses can activate biopolymers for structural identification in tandem mass spectrometry. Unlike conventional activation techniques where energy is incrementally deposited into the molecule to induce statistical dissociation of the most labile bonds [6], femtosecond pulse interactions yield a higher proportion of charged fragments and can induce non-statistical pathways which provide more complete structural information [7,8]. In addition, the amplitude, phase and polarisation of individual wavelength components of femtosecond pulses can be controlled $[9,10]$. This can be used to stimulate particular quantum pathways in complex molecules and the production of specific fragments can be optimized with pulse feedback control loops [11].

The main reason why femtosecond lasers have not been applied more widely to date is due to the cost and the technical ability required for their operation. However, commercial high power titanium:sapphire femtosecond laser systems are continually reducing in cost and size, and are moving towards turnkey operation. Ultimately, widespread application in mass spectrometry is likely to be based on fibre lasers which are more stable and can already produce femtosecond pulses with 10s of microjoules of energy at pulse rates approaching $\mathrm{MHz}$ [12].

The study of the mechanisms which govern the production of fragment ions from complex molecules in a strong laser field is still at a nascent stage. At present there is no working theoretical code which is capable of describing the interaction of a strong field with a complex molecule and the resulting fragmentation. However, non-adiabatic quantum electron dynamics approaches have been applied to large molecules with many de-localised electrons $[13,14]$, while techniques based on time dependent density functional theory are in development [15]. Meanwhile experimental work on biomolecular species is progressing $[7,8,16-29]$, although it is difficult to compare different studies due to variations in the initial conditions and the instrumentation used, e.g. protonation of the molecule, 
whether cooling gas is present, and the time window over which the dissociation is observed in the mass spectrometer.

When exposed to the strong field of a laser pulse, molecular orbitals are polarized which results in dynamic distortion of the molecule's potential energy landscape. This can soften bonds leading to dissociation processes which have been extensively studied in diatomic molecules [30,31]. In most cases any dissociation is preceded by multi-photon or tunnelling ionization. The ionization step is a necessary pre-cursor both to weaken the bond but also to excite vibrations in the cation due to FranckCondon transitions from the ground vibrational state of the neutral. Due to coupling with higher electronic states, the probability of dissociation by strong field bond softening is very dependent on the vibrational states populated in the resulting wavepacket and the evolving bond length.

The initial ionization site depends on the binding energy and polarizability of the orbitals, and if there is enhancement from intermediate states. For instance the chromophores of aromatic amino acids can be ionized preferentially by exploiting absorption bands in the wavelength range $250-280 \mathrm{~nm}$ to perform resonantly enhanced $1+1$ photon ionization at relatively low intensities using the $3^{\text {rd }}$ harmonic of a Ti:sapphire laser [18]. At longer wavelengths, ionization can proceed via non-resonant multiphoton absorption with typically six $800 \mathrm{~nm}$ photons required to ionise the lowest lying orbitals in biological molecules. At high intensities tunnelling becomes the dominant ionization mechanism, but it is difficult to predict the point at which this mechanism starts to dominate. The Keldysh adiabaticity parameter [32] is often used to identify this transition but is strictly only applicable for a zero range potential, so its use for extended, polarizable molecular systems is questionable [33,34]. If tunnelling dominates, ionization of the most weakly bound electron is usually favoured, although this is dependent on the polarizability [35], and bonding symmetry of the orbital [36]. The latter can result in a lower probability for ionisation of the highest occupied orbital compared to more strongly bound orbitals, which leads to specific dissociation products [37]. 
The initial radical charge site weakens neighbouring bonds which in combination with the strong laser field can lead to $\alpha$-cleavage reactions [21]. However, if energetically favourable, this radical can migrate to another part of the molecule, stimulating fragmentation at a site distant from the ionization. For instance, transfer of $\mathrm{H}$ atoms as far as seven amino acid residues along the peptide backbone have been observed in electron capture dissociation [38], producing full or partial side chain losses.

Alternatively, a positive hole can migrate through the molecule, which is an important phenomenon in fundamental biological processes [39-41]. In ground-breaking experiments on small peptides, Schlag, Weinkauf and co-workers were able to show that transfer of a charge from an ionization site at the Cterminal end of the molecule to the N-terminal can be controlled through selective absorption of an additional photon [20]. Schlathölter and co-workers also observed migration of charge towards lower energy orbitals following single photon ionisation of the peptide backbone, where the final destination of the charge led to characteristic fragmentation [42]. Recently, the timescale of such a process has been confirmed to be faster than any other observed to date in a biological molecule $[43,44]$, with theoretical predictions suggesting that even faster, coherent electron oscillations underlie this phenomenon [45-47].

By contrast, with conventional activation techniques the initial excitation energy is redistributed throughout the vibrational modes of the molecule on picosecond to nanosecond timescales, with statistical dissociation of labile bonds proceeding much more slowly [6]. In collision induced dissociation (CID), energy is inputted incrementally via multiple low energy collisions. Attachment of a mobile proton to a backbone nitrogen causes the $\mathrm{C}-\mathrm{N}$ peptide bond to be weakened, and if sufficient vibrational energy is localised here then the bond is broken. This may occur within microseconds to milliseconds in small molecules, but for large molecules with many degrees of freedom the dissociation rate is often too slow to be observed.

In a number of experiments by Dantus and Reid, a technique known as femtosecond laser induced dissociation (fs-LID) has been investigated $[7,8,16,17]$. They have studied a range of singly protonated peptides which were confined in a Paul trap and irradiated with $30 \mathrm{fs}, 800 \mathrm{~nm}$ pulses with 
intensities between $10^{13}-10^{14} \mathrm{Wcm}^{-2}$. After the molecules were irradiated with multiple laser pulses, the resulting fragments were mass analyzed. The precursor for most fragment ions was found to be the doubly charged distonic cation produced from single ionization which yields a more comprehensive set of characteristic sequence ions than other methods. While CID predominantly cleaves the peptide C-N bonds (defined as $b$ and $y$-type ions in the Roepstorff nomenclature [48]) and electron capture/transfer dissociation results in $c$ and $z$ ions from $\mathrm{N}-\mathrm{C}_{\alpha}$ fracture, fs-LID produces all backbone ion types and thus more complete sequence information. For instance for the peptide angiotensin II, 23 out of 42 possible $a, b, c, x, y, z$ sequence ions were produced [7]. As non-labile bonds are also broken, this technique can be used for determining sites of post-translational modifications and sequencing peptides with crosslinks $[8,17]$. In these experiments, ions were confined for hundreds of milliseconds, where a cooling gas was used to stabilise the product ions. In this way, both fast and slow dissociation processes were detected. Fragmentation could therefore be stimulated by a combination of the protonation of the molecule, the radical site generated by the ionisation, and absorption of additional photons by the molecule.

In this paper we have disentangled the role of fast and slow dissociation processes induced by femtosecond laser irradiation of neutral amino acids and peptides and determined the origin and importance of radical induced mechanisms. This was achieved by using a novel ion source in which neutral molecules were produced through laser induced acoustic desorption and subsequently ionized with an intense femtosecond laser. Mass spectra were obtained from an instrument operated in time of flight or ion trap mode, from which fragment ions useful for structural analysis are identified and the principal dissociation mechanisms deduced.

\section{Experimental Methods}

The experimental apparatus has been described in detail in previous papers $[49,50,51]$, so only a brief overview is given here. The femtosecond laser pulses used in this study were obtained from three different titanium:sapphire lasers operating with a fundamental wavelength in the range $790-800 \mathrm{~nm}$ : 
the Artemis laser facility [52] at the STFC Central Laser Facility (UK), a commercial Coherent ${ }^{\circledR}$ Libra laser, and an in-house laser system. The Artemis laser was used to produce pulses of 10-35 fs duration, while the two other lasers produced bandwidth limited pulses of $100 \mathrm{fs}$ duration. Third harmonic $(267 \mathrm{~nm})$ pulses of $100 \mathrm{fs}$ duration were generated by coupling the Libra laser to a Coherent ${ }^{\circledR}$ harmonic generation system (HGS).

Amino acid and peptide samples were obtained from Sigma-Aldrich and used for these studies without further purification. A $\mu \mathrm{g}$ quantity of the sample powder was applied to a $10 \mu \mathrm{m}$ thick tantalum foil which was clamped onto a stainless steel holder and inserted into the repeller electrode of a mass spectrometer of our own design (KEIRAlite). This instrument could be operated as a conventional linear time of flight (ToF) or as a high resolution electrostatic ion trap [49]. The uncoated side of the foil was irradiated with a $5 \mathrm{~ns}, 355 \mathrm{~nm}, 0.2 \mathrm{~mJ}$ laser pulse focused to an intensity of approximately $3 \times 10^{8} \mathrm{Wcm}^{-2}$ to generate an acoustic wave which volatilizes the sample on the other side of the foil [51]. The plume was allowed to evolve for $15 \mu$ s before irradiation with the femtosecond laser. The femtosecond pulses were focused using a spherical lens with a focal length of $15 \mathrm{~cm}$. The beam intersected the plume approximately $10 \mathrm{~mm}$ before reaching the focal point, at a distance of about $2 \mathrm{~mm}$ from the sample foil surface. The interaction intensity was calibrated from the plotting the yield of $\mathrm{Xe}^{+}$ions as a function of the laser pulse energy to determine the saturation intensity.

In ToF mode, ions generated from the interaction were accelerated into a $0.5 \mathrm{~m}$ flight tube with an energy of $5 \mathrm{keV}$. The signal from a channel electron multiplier was pre-amplified and recorded with $2.5 \mathrm{~ns}$ resolution, giving an overall resolving power of $m / \Delta m \sim 150$. Following ionization by the laser pulse, there is a time window of about 20 ns within which any dissociation must occur for the fragment ion masses to be correctly analysed. Slower processes result in significant movement of the parent ion prior to dissociation which produces longer fragment flight times than expected. In trapping mode, potentials were applied to an additional set of electrodes which mirrored the ion 
extraction potentials. With the appropriate potential applied to two focussing lenses, ions were trapped on stable trajectories and over time separated into different ion bunches depending on their mass to charge ratio. Signals from these ion bunches were acquired from the image charge induced on a pickup electrode and mass spectra were obtained using the frequency analysis algorithm CHIMERA which provided a resolving power of up to $10^{4}[49]$.

\section{Results and Discussion}

To understand how the strong electric field of an intense, femtosecond laser pulse acts on a whole molecule, it is often of benefit to first investigate how less complicated, individual building blocks behave under similar conditions. Therefore, experimental investigations of amino acids are a good starting point for understanding the femtosecond laser induced dissociation of peptides. As nonresonant multi-photon or tunnelling ionisation preferentially ionizes the most weakly bound electrons, in isolated amino acids ionization will most probably remove an electron from the nitrogen lone pair of the amino group ( $\mathrm{n}_{\mathrm{N}}$ orbital) or the highest occupied orbital of the functional side chain group $\left(\pi_{1}\right)$. The vertical ionization energy of the former is around $8.5 \mathrm{eV}$ for most amino acids [20], while more weakly bound electrons can be found in some of the side chain groups (see Table 1). When incorporated into a peptide chain, the ionization energy of the nitrogen lone pair is increased by at least $0.5 \mathrm{eV}$, but is almost unchanged for the nitrogen in the $\mathrm{N}$-terminal residue.

\begin{tabular}{|c|c|c|c|}
\hline Amino Acid & 3 letter code & $\begin{array}{c}\text { Ionization } \\
\text { Energy (eV) }\end{array}$ & HOMO location \\
\hline$\alpha$-Alanine & Ala & 8.6 & N lone pair \\
\hline Phenylalanine & Phe & 8.4 & Phenyl group/N lone pair \\
\hline Tyrosine & Tyr & 8.0 & Phenol group \\
\hline Histidine & His & 7.7 & Imidazole group \\
\hline
\end{tabular}

Table 1. Vertical ionization potentials for some amino acids [53] 
Mass spectra produced from the interaction of intense $\left(10^{13} \mathrm{Wcm}^{-2}\right)$, femtosecond $(100 \mathrm{fs}), 800 \mathrm{~nm}$ laser pulses with the neutral amino acids alanine, phenylalanine, tyrosine, and histidine are shown in Figure 1. The insets in each figure show the NIST electron impact ionization mass spectra for these molecules for comparison. It can be seen that parent peaks are much more prominent in the femtosecond laser spectra than for electron impact. Major fragment ions which correspond to cleavage of distinct groups from the molecule can also be readily identified in the laser spectra. Other mass fragments are also present which are produced from multiple bond cleavages. These become dominant at higher intensities $\left(10^{14} \mathrm{Wcm}^{-2}\right)$ where there is substantial coupling of additional energy into the molecule via further excitation or ionization which results in more extensive fragmentation.

Alanine has the highest ionization potential of all the amino acids we studied. Removal of an electron from the $\mathrm{n}_{\mathrm{N}}$ orbital in alanine stimulates alpha cleavage of the $\mathrm{C}_{\alpha}-\mathrm{C}$ bond producing the immonium ion $\left([\mathrm{M}-\mathrm{COOH}]^{+}\right)$which dominates the spectrum in Fig. 1a. In contrast, for tyrosine (Fig. 1c) and histidine (Fig. 1d) the most weakly bound electron is in a side chain orbital and the dominant peaks are due to complete side chain ions, $R^{+}\left(107 \mathrm{u}\right.$ and $81 \mathrm{u}$ respectively) produced from $\mathrm{C}_{\alpha}-\mathrm{C}_{\beta}$ cleavage.

Phenylalanine is an interesting intermediate case where the $\mathrm{n}_{\mathrm{N}}$ orbital and $\pi_{1}$ state of the phenyl group have similar binding energies. The spectrum shows that the main fragments are associated with charge on the amino group causing $\mathrm{C}_{\alpha}-\mathrm{C}(120 \mathrm{u})$ and $\mathrm{C}_{\alpha}-\mathrm{C}_{\beta}(74 \mathrm{u})$ cleavages. Charge localised on the amino group is either produced directly from the initial ionization of the $\mathrm{n}_{\mathrm{N}}$ orbital or results from transfer of the charge after ionisation of the phenyl $\pi_{1}$ state. It is interesting to note that the spectrum obtained from using $267 \mathrm{~nm}$ to selectively ionize the $\pi_{1}$ orbital (Figure 5 of reference [51]), produces a similar spectrum to Figure 1(b). This suggests that there is a high probability of the charge being transferred to the amino group prior to dissociation. Indeed it has recently been shown that the timescale for intramolecular charge migration to the amino group in phenylalanine is about $30 \mathrm{fs}$ [43]. 


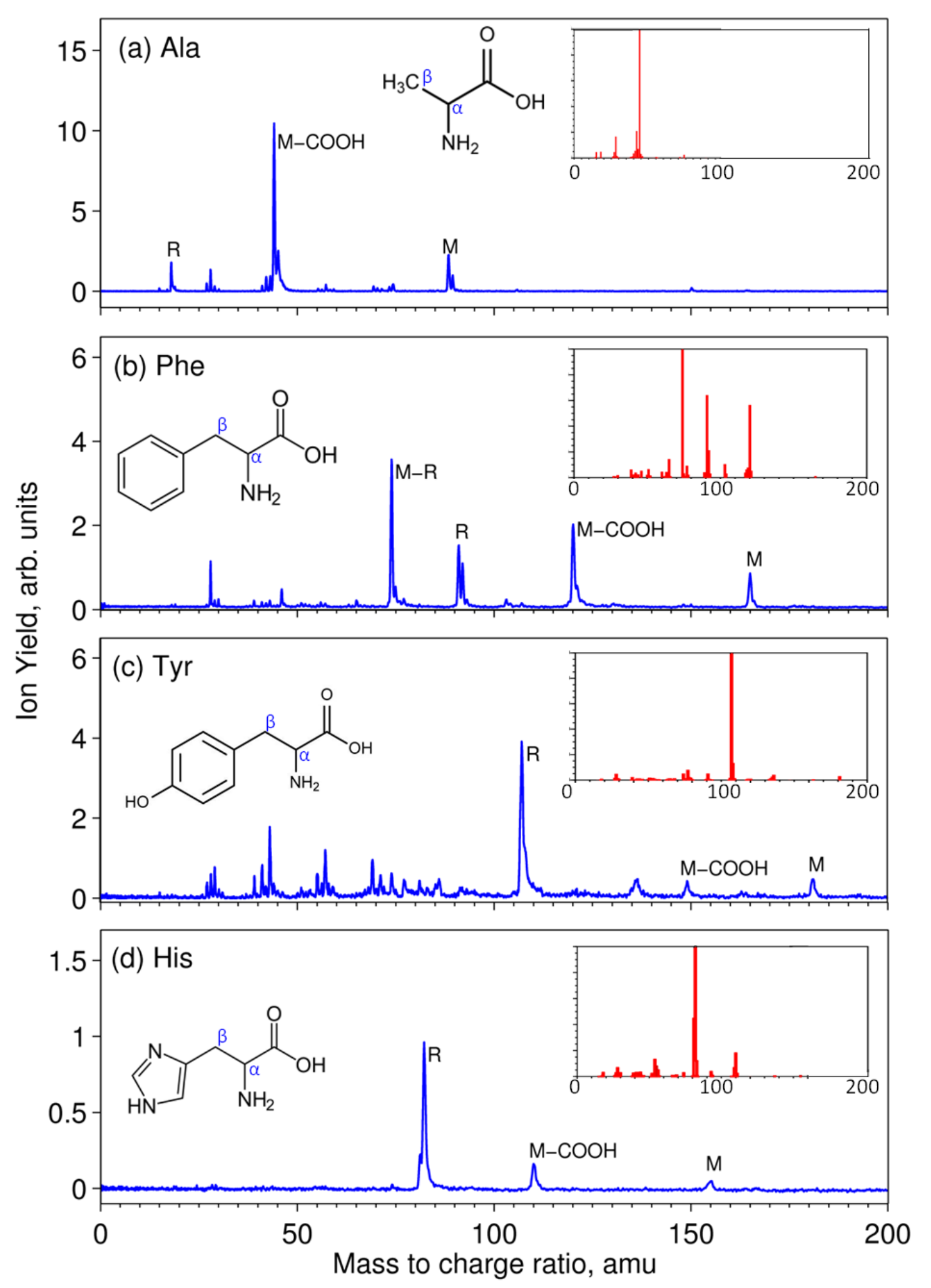

Figure 1. Spectra obtained with $100 \mathrm{fs}, 800 \mathrm{~nm}$ pulses at an intensity of $10^{13} \mathrm{Wcm}^{-2}$ for amino acids: (a)

Alanine; (b) Phenylalanine; (c) Tyrosine; (d) Histidine. $\mathrm{M}$ is the parent molecular ion; $(\mathrm{M}-\mathrm{COOH})$ immonium ion; R side chain ion; $(\mathrm{M}-\mathrm{R})$ side chain group loss. The insets present NIST electron impact ionization spectra for each of the molecules [54]. 
These spectra are similar to other studies which used UV and VUV wavelengths for one or two photon ionization of these molecules [21,55,56,57]. This suggests that it is the ionization itself (and any subsequent charge migration) which is the critical stimulus for fragmentation, provided the cation possesses several $\mathrm{eV}$ of additional energy to break a bond. This additional energy can partly arise from differences in the vertical and adiabatic ionization potentials (typically $1 \mathrm{eV}$ or more [53]), the ionized electron transferring some excess energy, or further photon absorption by the cation. In contrast, a previous study of fs-LID of protonated amino acids [16] yielded very different spectra compared to those obtained in Figure 1. This is not surprising as the presence of the proton increases the ionization energy by an amount which depends on the physical proximity of the proton to the orbital. Hence a higher laser intensity is required for laser induced dissociation $\left(7.5 \times 10^{13} \mathrm{Wcm}^{-2}\right)$. For non-basic amino acids (e.g. alanine, phenylalanine, tyrosine) the proton is attached to the amino group which strongly suppresses ionization of the $\mathrm{n}_{\mathrm{N}}$ orbital, while in histidine the proton binds to the basic imidazole preventing ionization of a weakly bound electron from this group. So unlike our results in Figure 1, there is no dissociation observed for protonated alanine and histidine in reference [17]. While our tyrosine spectrum is dominated by side chain ions $(107 \mathrm{u})$, for the protonated species this fragment is much smaller, whereas loss of the carboxyl group following ionization of the phenol group to form a doubly charged immonium ion is prominent.

Figure 2 presents photoionization spectra at $800 \mathrm{~nm}$ for the four small peptides His-Gly, Ala-Phe, Phe-Ala, and Leu-Leu-Tyr. Also, shown in Figure 3 are spectra obtained at $267 \mathrm{~nm}$ for the peptides with Phe and Tyr chromophores. For all these spectra, apart from Phe-Ala at $800 \mathrm{~nm}$, the parent ion (M) can be clearly identified. The proportion of parent ions is larger for the $267 \mathrm{~nm}$ photoionization as the photon absorption is resonantly enhanced allowing a much lower intensity to be used. In Figure 3(d) it can be seen that for very low intensities only parent ions are produced for Leu-Leu-Tyr, which establishes that the any molecular fragmentation is initiated by the femtosecond pulses and not by the desorption process. However, it should be noted that the molecules are not produced at room 
temperature [31] and therefore will have a degree of vibrational excitation which will lower dissociation barriers.

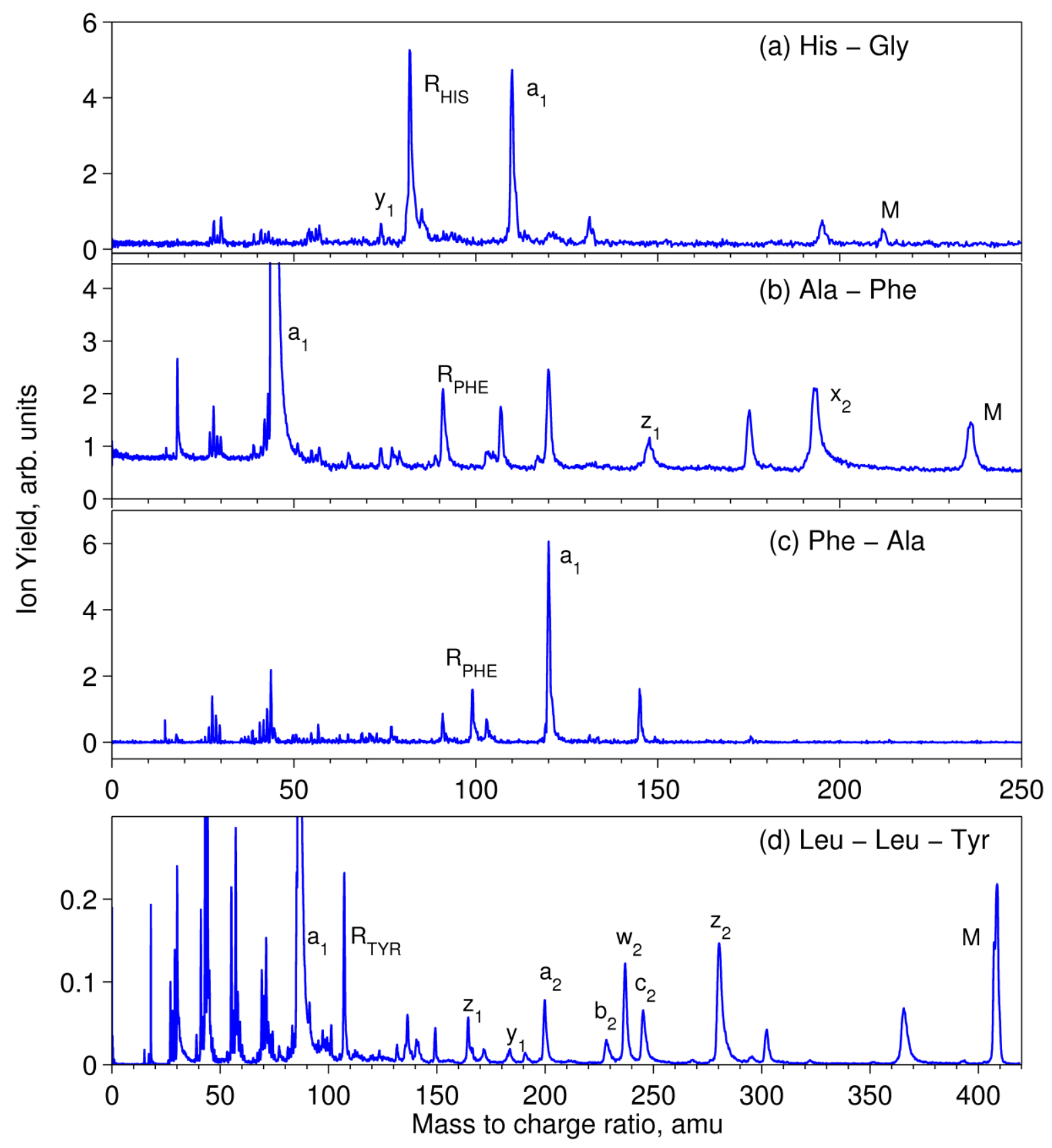

Figure 2. Spectra obtained at $800 \mathrm{~nm}$ for peptides: (a) His-Gly at an intensity of $1.5 \times 10^{13} \mathrm{~W} \mathrm{~cm}^{-2}$; (b) Ala-Phe at $8 \times 10^{12} \mathrm{~W} \mathrm{~cm}^{-2}$; (c) Phe-Ala at $1.0 \times 10^{13} \mathrm{~W} \mathrm{~cm}^{-2}$ all with $100 \mathrm{fs}$ pulses; (d) Leu-Leu-Tyr at $5 \times 10^{12} \mathrm{~W} \mathrm{~cm}^{-2}$ with 15 fs pulses.

The most noticeable feature of all these spectra is that while Phe, Tyr and His residues generate prominent side chain ions $\left(\mathrm{R}^{+}\right)$due to their low ionization potentials, $\mathrm{N}$-terminal immonium ions $\left(a_{1}\right.$ 
ions) generally dominate the spectra. Indeed at $800 \mathrm{~nm}$ these immonium ion yields are even stronger than those obtained for the isolated amino acids, suggesting there is additional charge migration to the $\mathrm{N}$-terminal following ionization in these peptides. It can be seen in Figure 3 that even with selective ionization of chromophores distant from the $\mathrm{N}$-terminal, the immonium ion yields are still significant. This confirms the conclusions made by Weinkauf et al. in studies using ns lasers that charge can efficiently migrate along the peptide backbone on ultrafast timescales [20].

The strong production of side chain ions in every spectrum provides an efficient means of identifying the presence of amino acids in a peptide chain after intense, femtosecond pulse irradiation of the neutral molecules. The immonium ions also allow direct identification of the $\mathrm{N}$-terminal residue, although for protonated peptides this could be suppressed if the proton is sited there. Various ions produced from backbone cleavages can also be seen in all the spectra, particularly at $800 \mathrm{~nm}$. The designation of these ions is shown in the Figure 2 spectra. For Leu-Leu-Tyr it can be seen that 7 out of the 12 possible backbone ions are present along with ions produced from Leu and Tyr side chain losses (see Figure 4). In contrast conventional CID is predicted to yield only 3 backbone ions $-y_{1}, y_{2}$, $b_{2}[58]$. 


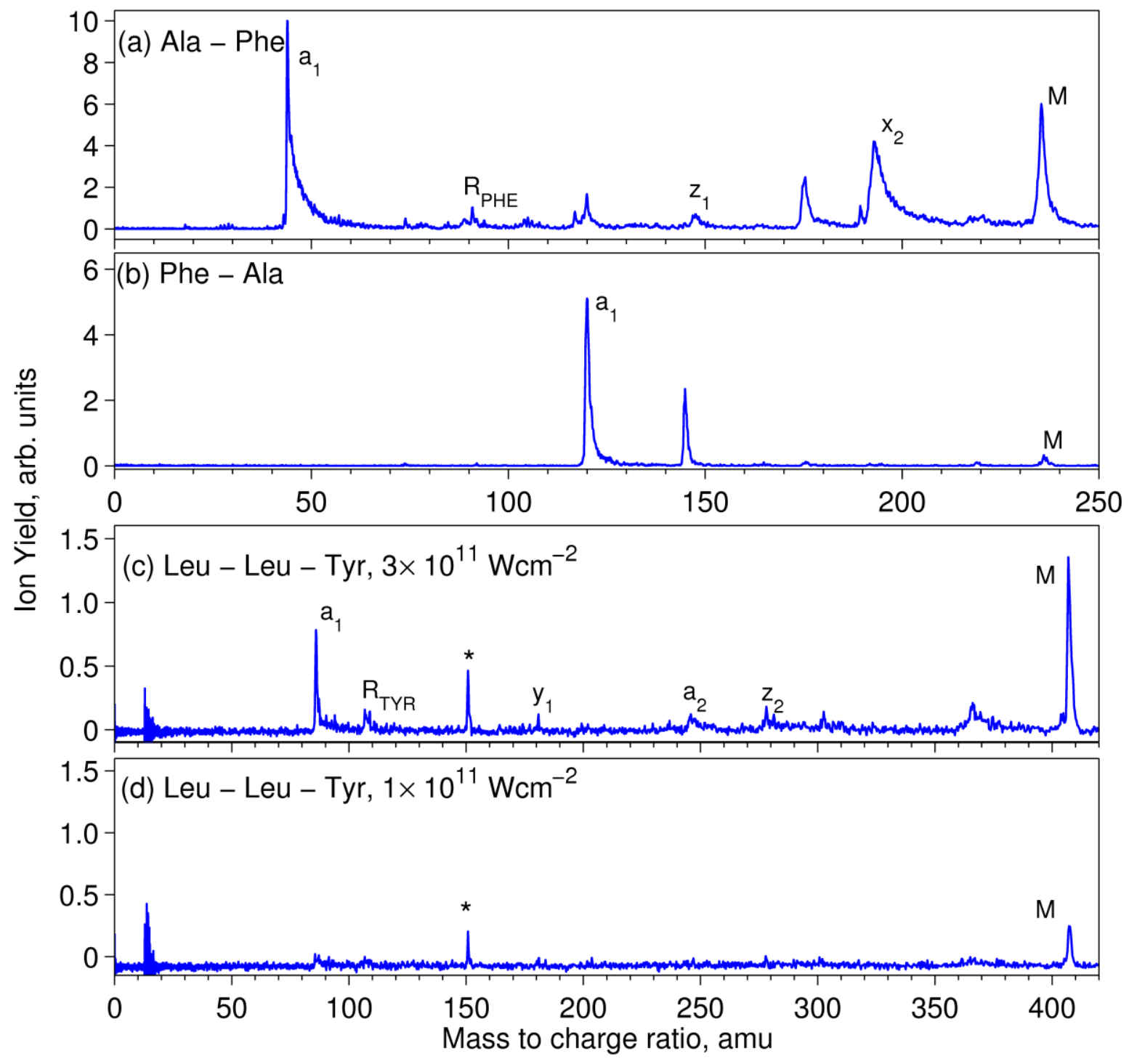

Figure 3. Spectra obtained with $100 \mathrm{fs}, 267 \mathrm{~nm}$ pulses for peptides: (a) Ala-Phe for an intensity of $2 \times 10^{11}$ $\mathrm{Wcm}^{-2}$; (b) Phe-Ala at $2 \times 10^{11} \mathrm{~W} \mathrm{~cm}^{-2}$; (c) Leu-Leu-Tyr at $3 \times 10^{11} \mathrm{~W} \mathrm{~cm}^{-2}$; (d) Leu-Leu-Tyr at $1 \times 10^{11} \mathrm{~W}$ $\mathrm{cm}^{-2}$ (peak labelled $*$ is the parent ion of acetaminophen which is a contaminant from an earlier experiment)

These results show that a significant number of structural ions can be produced without the molecule being initially protonated. The immonium and side chain ions which have the greatest yields are due to the positive hole produced from the initial ionization or subsequent ultrafast charge migration. Meanwhile, most of the backbone ions have masses which are $1 \mathrm{u}$ more than that expected from a simple breakage suggesting that in this case the radical induced cleavage is facilitated by $\mathrm{H}$ atom migration. 


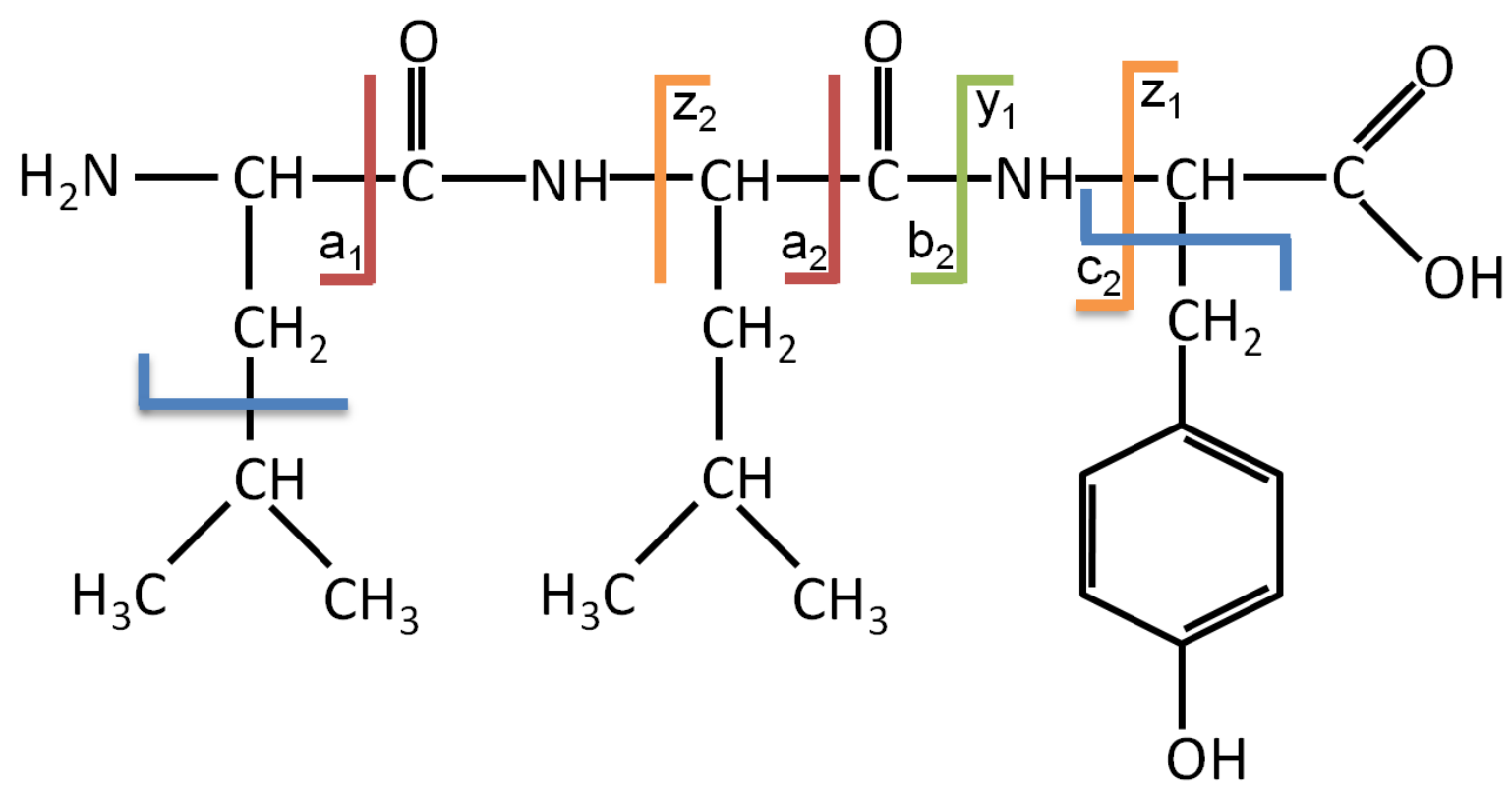

Figure 4. Fragment ions of Leu-Leu-Tyr observed in Figure 2(d).

Fast dissociation is necessary for fragment ions to be observed in our instrument, meaning that most of the fragmentation is non-statistical. However, there may be still some contribution from statistical fragmentation for channels with low activation barriers. When the molecule is given substantial additional energy statistical processes become relatively more important. This was achieved by using higher intensities at $267 \mathrm{~nm}$ so that further photons are absorbed by the chromophore. Figure 5(a) shows ToF mass spectra of Phe-Ala obtained at $267 \mathrm{~nm}$ and a maximum intensity of $5 \times 10^{12} \mathrm{~W} \mathrm{~cm}^{-2}$ where tails in the peaks are a result of significant fragmentation in the acceleration region. These ions acquire a lower final velocity than would be obtained from prompt dissociation, corresponding to a decay time of around a few hundred nanoseconds. By contrast, the ion trap spectrum in Figure 5(b) is acquired from the signal obtained from trapping the ions for 10 ms. If Figures 5 (a) and (b) are compared it can be seen that the parent ion and some fragment ions are absent from the ion trap spectrum indicating that these ions are undergoing dissociation within around $1 \mathrm{~ms}$. 


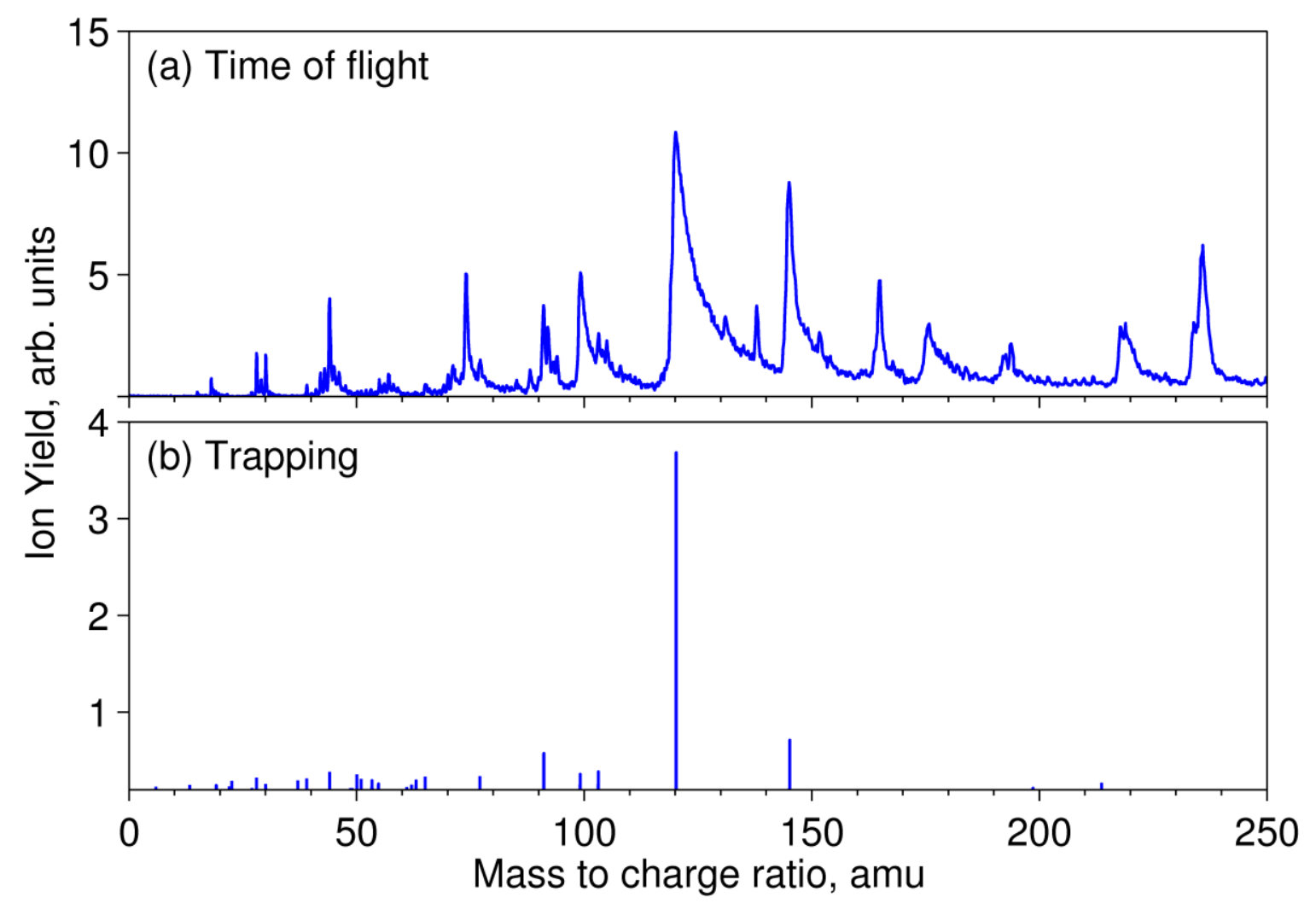

Figure 5. Phe-Ala spectra obtained with $267 \mathrm{~nm}, 100 \mathrm{fs}$ pulses and an intensity of $5 \times 10^{12} \mathrm{~W} \mathrm{~cm}^{-2}$ acquired in (a) ToF and (b) trapping modes.

This demonstrates the importance of the observational time window in determining whether statistical or non-statistical processes are dominant. For many activation techniques both mechanisms will be present, so the measured outcome depends on the instrumentation employed. For instance it is interesting to compare our results for Leu-Leu-Tyr (experiment I), with previous work using nanosecond laser pulses by Weinkauf et al. [19,20] (IIa,IIb) who also used a ToF device, and Cui et al. [22] (III) who used a quadrupole ion trap. When experiment III was configured to detect prompt fragmentation processes only, the $267 \mathrm{~nm}$ photoionization spectra from I, IIa, and III appear to be in good mutual agreement, with fast charge migration to the $\mathrm{N}$-terminal producing leucine immonium ions. However, there is a discrepancy in the two color experiments IIb and III, where soft photoionization with low intensity $267 \mathrm{~nm}$ pulses was followed by a 532nm pulse which is resonantly absorbed by the chromophore cation. In experiment III, absorption of one green photon was sufficient to move the charge from the chromophore to the $\mathrm{N}$-terminal. This produced immonium ions in 
experiment III, but none were observed in experiment IIb. This discrepancy is due to the millisecond observation time available in III which allowed a slow statistical dissociation process to be measured, while in experiment IIb the low detection efficiency of these events (about 1 in every 1000) meant that statistical dissociation processes were not observed [59].

\section{Conclusion}

Due to the complexity of the dynamics, understanding how intense, femtosecond lasers interact with biological molecules is a challenging proposition. To date there has been little theoretical work and only a handful of experiments performed on biomolecules in the gas phase. With a wide range of possible mechanisms and timescales contributing to fragment ion production, elucidating the relative importance of each process from experimental mass spectra is difficult. In this study we have simplified the problem by studying only neutral target molecules and focussing mainly on ions from fast fragmentation. Consequently, our results have highlighted non-ergodic radical driven dissociation processes.

For amino acids we found that the initial ionization site largely determines which fragment ions dominate the spectrum. In a strong field, ionization is preferentially from the most weakly bound molecular orbitals which are usually the electron lone pair on the $\mathrm{N}$ atom of the amino group or the highest occupied molecular orbital of the side chain group. This tends to produce immonium or side chain ions via a fast alpha cleavage mechanism. For small peptides we found even higher yields for the immonium ions of $\mathrm{N}$-terminal residues which we have attributed to ultrafast charge migration to the N-terminal preceding any dissociation. A range of less prominent, but structurally important ions, were also produced which appear to originate from radical initiated dissociation following $\mathrm{H}$ atom migration.

Although our apparatus was optimised for observing fast dissociation processes, we also obtained evidence for the contribution of statistical processes. This was most evident in spectra obtained with high intensity $267 \mathrm{~nm}$ pulses, where long tails on the peaks in the time of flight spectrum were due to delayed dissociation in the ion acceleration region. Spectra obtained from the trapping mode also had 
an absence of parent and some fragment ions showing that these ions underwent unimolecular dissociation within a millisecond.

Overall this work shows that fast radical induced dissociation is a major contributor to extensive breakage of non-labile bonds and production of sequence ions. Differences in the initial molecular target and experimental set-up make direct comparison of the present work with pioneering studies by Dantus and Reid $[7,8,16,17]$ difficult. However, we have shown that extensive structural information can be obtained without protonation of the molecule and our results for Leu-Leu-Tyr at resonant UV wavelengths are in good agreement with other studies using this neutral tri-peptide $[19,22]$, albeit we used considerably shorter pulse lengths.

Although the use of femtosecond lasers in mass spectrometry to date has been limited to specialised research groups, these proof-of-principal experiments have demonstrated their potential as efficient ionization and activation sources. In the present work, with the appropriate pulse length, intensity, and wavelength it is possible to generate parent radical cations of small biological molecules along with fragment ions which yield substantial structural information. Other studies have even demonstrated that ion yields and photoelectron spectra produced by femtosecond pulses could also be used to determine the chirality of such molecules $[60,61]$. Therefore, as the cost, size, and complexity of femtosecond laser technology reduces, commercial mass spectrometry applications are increasingly likely in the next few years.

\section{Acknowledgements}

This work was supported by the Leverhulme Trust, STFC through access to the Artemis Laser Facility, and EPSRC through the STFC Laser Loan Pool. CRC acknowledges support from EPSRC through the Postdoctoral Fellowship Programme (Grant Number EP/H027319/1) and LB and MJD acknowledge the support of the Department of Employment and Learning, Northern Ireland. TJK and JTC acknowledge support from Science Foundation Ireland. We also acknowledge the technical assistance provided by Phil Rice at the Artemis Laser Facility. 


\section{References}

[1] Brodbelt, J.S. Shedding Light on the Frontier of Photodissociation, . J. Am. Soc. Mass Spectrom. 2011, 22 , 197-206

[2] Kelly, O.; Duffy, M.J; King, R.B.; Belshaw, L.; Williams, I.D.; Sa, J.; Calvert C.R.; Greenwood, J.B.; Femtosecond lasers for mass spectrometry: Proposed application to catalytic hydrogenation of butadiene. Analyst 2012, 137, 64-69

[3] Peng, J.; Puskas, N.; Corkum, P.B.; Rayner, D.M.; Loboda, A.V.; High-Pressure Gas Phase Femtosecond Laser Ionization Mass Spectrometry. Anal. Chem. 2012, 84, 5633-5640

[4] Chang, Y-C.; Imasaka, T.; Simple Pretreatment Procedure Combined with Gas Chromatography /Multiphoton Ionization/Mass Spectrometry for the Analysis of Dioxins in Soil Samples Obtained after the Tohoku Earthquake. Anal. Chem. DOI: 10.1021/ac3028917

[5] Watanabe-Ezoe, Y.; Li, X.; Imasaka, T.; Uchimura, T.; Imasaka, T. Gas Chromatography/Femtosecond Multiphoton Ionization/Time-of-Flight Mass Spectrometry of Dioxins. Anal. Chem. 2010, 82, 6519-6525

[6] Sleno, L.; Volmer, D.A. Ion activation methods for tandem mass spectrometry. J. Mass Spectrom. 2004; 39 , 1091-1112

[7] Kalcic, C. L.; Gunaratne, T.; Jones, A. D.; Dantus, M.; Reid, G. E. Femtosecond Laser-induced Ionization/ Dissociation of Protonated Peptides. J. Am. Chem. Soc. 2009, 131, 940-942.

[8] Zhu, X.; Kalcic,C.L.; Winkler, N.; Lozovoy, V.V; Dantus, M.; Applications of Femtochemistry to Proteomic and Metabolomic Analysis, J. Phys. Chem. A 2010, 114, 10380-10387

[9] Brixner, T.; Gerber, G. Quantum Control of Gas-Phase and Liquid-Phase Femtochemistry. ChemPhysChem 2003, 4, 418-438

[10] Lozovoy, V.V; Zhu, X.; Gunaratne, T.C.; Harris, D.A.; Dantus, M.; Control of Molecular Fragmentation Using Shaped Femtosecond Pulses. J. Phys. Chem. A 2008, 112, 3789-3812

[11] Laarmann, T.; Shchatsinin, I.; Singh, P.; Zhavoronkov, N.; Schulz, C.P.; Hertel, I.V. Femtosecond pulse shaping as analytic tool in mass spectrometry of complex polyatomic systems. J. Phys. B: At., Mol. Opt. Phys., 2008, 41, 074005

[12] Zhang, X.S.; Schneider, E.; Taft, G.; Kapteyn, H.; Murnane, M.; Backus, S. Multi-microjoule, MHz repetition rate Ti:sapphire ultrafast regenerative amplifier system, Optics Express 2012, 20, 7015-7021,

[13] Lezius, M.; Blanchet, V.; Ivanov, M.Y.; Stolow, A. Polyatomic molecules in strong laser fields: Nonadiabatic multielectron dynamics. J. Chem. Phys., 2002, 117, 1575-1588

[14] Markevitch, A.N.; Romanov, D. A.; Smith, S.M.; Schlegel, H.B.; Ivanov, M.Y.; Levis, R.J. Sequential nonadiabatic excitation of large molecules and ions driven by strong laser fields. Phys. Rev. A 2004, 69, 013401 [15] Dundas, D. Multielectron effects in high harmonic generation in N2 and benzene: simulation using a nonadiabatic quantum molecular dynamics approach for laser-molecule interactions. arXiv:1202.0674v3

[16] Kalcic, C. L.; Reid, G. E.; Lozovoy, V.V; Dantus, M. Mechanism Elucidation for Nonstochastic Femtosecond Laser-Induced Ionization/Dissociation: From Amino Acids to Peptides. J. Phys. Chem. A 2012, $116,2764-2774$

[17] Smith, S.A.; Kalcic, C. L.; Safran, K.A.; Stemmer, P.M.; Dantus, M.; Reid, G. E. Enhanced Characterization of Singly Protonated Phosphopeptide Ions by Femtosecond Laser-induced Ionization/Dissociation Tandem Mass Spectrometry (fs-LID-MS/MS). J. Am. Soc. Mass Spectrom. 2010, 21, 2031-2040

[18] Weinkauf, R.; Aicher, P.; Wesley, G.; Grotemeyer, J.; Schlag, E.W. Femtosecond Versus Nanosecond Multiphoton Ionization and Dissociation of Large Molecules. J. Phys. Chem. 1994, 98, 8381-8391.

[19] Weinkauf, R.; Schanen, P.; Yang, D.; Soukoura, S.; Schlag, E.W. Elementary Processes in Peptides: Electron Mobility and Dissociation in Peptide Cations in the Gas Phase. J. Phys. Chem. 1995, 99, 11255- 11265

[20] Weinkauf, R.; Schanen, P.; Metsala, A.; Schlag, E.W.; Bürgle M.; Kessler, H.; Highly Efficient Charge Transfer in Peptide Cations in the Gas Phase: Threshold Effects and Mechanism. J. Phys. Chem. 1996, 100, $18567-18585$.

[21] Vorsa, V.; Kono, T.; Willey, K. F.; Winograd, N. Femtosecond Photoionization of Ion Beam Desorbed Aliphatic and Aromatic Amino Acids: Fragmentation via $\alpha$-Cleavage Reactions. J. Phys. Chem. B 1999, 103, 7889-7895.

[22] Cui, W.; Hu, Y.; Lifshitz, C. Time resolved photodissociation of small peptide ions. Eur. Phys. J. D 2002, $20,565-571$ 
[23] Tembreull, R.; Lubman, D.M. Resonant Two-Photon Ionization of Small Peptides Using Pulsed Laser Desorption in Supersonic Beam Mass Spectrometry. Anal. Chem. 1987, 59, 1003-1006

[24] Lockyer, N.; Vickerman, J.C. Multiphoton ionization mass spectrometry of small biomolecules with nanosecond and femtosecond laser pulses. Int. J. Mass Spectrom. 1998, 176, 77-86

[25] Lockyer, N.; Vickerman, J.C. Single photon and femtosecond multiphoton ionisation of the dipeptide valylvaline. Int. J. Mass Spectrom. 2000, 197, 197-209

[26] Willingham, D.; Kucher, A.; Winograd, N. Strong-field ionization of sputtered molecules for biomolecular imaging. Chem. Phys. Lett. 2009, 468, 264-269

[27] Grégoire, G.; Dedonder-Lardeux, C.;Jouvet, C.; Desfrancois, C.; Fayeton, J.A. Ultrafast excited state dynamics in protonated GWG and GYG tripeptides. Phys. Chem. Chem. Phys., 2007, 9, 78-82

[28] Pérot, M.; Lucas, B.; Barat, M.; Fayeton, J.A.; Jouvet, C. Mechanisms of UV Photodissociation of Small Protonated Peptides. J. Phys. Chem. A 2010, 114, 3147-3156

[29] Kelly, O.; Calvert, C.R.; Greenwood, J.B.; Zettergren, H.; Nielsen, S.B.; Wyer, J.A. Effects of Charge Location on the Absorptions and Lifetimes of Protonated Tyrosine Peptides in Vacuo. J. Phys. Chem. A 2012, 116, 1701-1709

[30] Posthumus, J. H. The dynamics of small molecules in intense laser fields. Rep. Prog. Phys. 2004, 67, 623665

[31] Calvert, C.R.; Bryan, W.A.; Newell, W.R.; Williams, I.D. Time-resolved studies of ultrafast wavepacket dynamics in hydrogen molecules. Phys. Rep. 2010, 491, 1-28

[32] Keldysh, L.V. Sov. Phys. JETP 1965, 20, 1307

[33] DeWitt, M.J.; Levis, R.J. Observing the Transition from a Multiphoton-Dominated to a Field-Mediated Ionization Process for Polyatomic Molecules in Intense Laser Fields. Phys. Rev. Lett. 1998, 81, 5101-5104 [34] DeWitt, M.J.; Levis, R.J. Calculating the Keldysh adiabaticity parameter for atomic, diatomic, and polyatomic molecules. J. Chem. Phys. 1998, 108, 7739-7742

[35] Hankin, S.M.; Villeneuve, D.M.; Corkum, P.B.; Rayner, D.M. Nonlinear Ionization of Organic Molecules in High Intensity Laser Fields. Phys. Rev. Lett. 2000, 84, 5082-5085

[36] Muth-Böhm, J.; Becker, A.; Faisal, F. H. M. Suppressed Molecular Ionization for a Class of Diatomics in Intense Femtosecond Laser Fields. Phys. Rev. Lett. 2000, 85, 2280-2283

[37] Boguslavskiy, A.E.; Mikosch, J.; Gijsbertsen, A.; Spanner, M.; Patchkovskii, S.; Gador, N.; Vrakking, M.J.J.; Stolow, A. The Multielectron Ionization Dynamics Underlying Attosecond Strong-Field Spectroscopies. Science 2012, 335, 1336-1340

[38] Savitski, M.M.; Nielsen M.L.; Zubarev, R.A. Side-Chain Losses in Electron Capture Dissociation To Improve Peptide Identification. Anal. Chem. 2007, 79, 2296-2302

[39] van Grondelle, R.; Novoderezhkin, V.I. Quantum effects in photosynthesis. Procedia Chemistry 2011, 3, $198-210$

[40] Cordes, M.; Giese, B. Electron Transfer in Peptides and Proteins. Chem. Soc. Rev. 2009, 38, 892-901

[41] Becker, D.; Adhikary, A.; Sevilla, M. D. The Role of Charge and Spin Migration in DNA Radiation Damage. Charge Migration in DNA; Chakraborty, T., Ed.; Springer: New York, 2007; pp 139-175.

[42] González-Magana, O.; Reitsma, G.; Bari, S.; Hoekstra, R.; Schlatöhlter, T. Length effects in VUV photofragmentation of protonated peptides. Phys. Chem. Chem. Phys., 2012, 14, 4351-4354

[43] Lehr, L.; Horneff, T.; Weinkauf, R.; Schlag, E. W. Femtosecond Dynamics after Ionization: 2-PhenylethylN,N-dimethylamine as a Model System for Nonresonant Downhill Charge Transfer in Peptides. J. Phys. Chem. A 2005, 109, 8074-8080.

[44] Belshaw, L.; Calegari, F.; Duffy, M.J.; Trabattoni, A.; Poletto, L.; Nisoli, M.; Greenwood, J.B. Observation of Ultrafast Charge Migration in an Amino Acid, J. Phys. Chem. Lett. 2012, 3, 3751-3754

[45] Cederbaum, L.S.; Zobeley, J. Ultrafast charge migration by electron correlation. Chem. Phys. Lett. 1999, 307, 205-210

[46] Remacle, F.; Levine, R. D. An Electronic Time Scale in Chemistry. Proc. Natl. Acad. Sci. U.S.A. 2006, 103, 6793-6798.

[47] Kuleff, A. I.; Lünnemann, S.; Cederbaum, L. S. Electron-Correlation-Driven Charge Migration in Oligopeptides. Chem. Phys. 2012, DOI: http://dx.doi.org/10.1016/j.chemphys.2012.02.019 .

[48] Roepstorff, P.; Fohlman, J. Proposal for a common nomenclature for sequence ions in mass spectra of peptides. Biomed Mass Spectrom. 1984, 11, 601

[49] Greenwood, J. B.; Kelly, O.; Calvert, C. R.; Duffy, M. J.; King, R. B.; Belshaw, L.; Graham, L.; Alexander, J.D.; Williams, I. D.; Bryan, W. A.; Turcu, I. C. E.; Cacho, C. M.; Springate, E. A comb-sampling method for enhanced mass analysis in linear electrostatic ion traps. Rev. Sci. Inst. 2011, 82, 043103

[50] Kelly, O.; Duffy, M. J.; King, R. B.; Belshaw, L.; Williams, I. D.; Sa, J.; Calvert, C. R.; Greenwood, J. B.; Femtosecond lasers for mass spectrometry: Proposed application to catalytic hydrogenation of butadiene.

Analyst 2012, 137, 64-69 
[51] Calvert C.R.; Belshaw, L.; Duffy, M.J; Kelly, O.; King, R.B.; Smyth, A.G.; Kelly, T.J.; Costello, J.T.; Timson, D.J.; Bryan, W.A.; et al. LIAD-fs Scheme for Studies of Ultrafast Laser Interactions with Gas Phase Biomolecules. Phys. Chem. Chem. Phys., 2012, 14, 6289-6297

[52] I. C. E. Turcu, et al., Proc. SPIE-Int. Soc. Opt. Eng. 2009, 7469, 746902.

[53] Schlag, E.W.; Sheu, S-Y.; Yang, D-H.; Selzle, H.L.; Lin, S.H.; Angwe. Chem. Int. Ed. 2007, 46, 3196-3210

[54] NIST electron impact ionisation spectra

[55] Wilson, K.R.; Belau, L.; Nicolas, C.; Jimenez-Cruz, M.; Leone, S.R.; Ahmed, M. Direct determination of the ionization energy of histidine with VUV synchrotron radiation. Int. J. Mass Spectrom. 2006, 249-250, 155161

[56] Jochims, H-W.; Schwell, M.; Chotin, J-L.; Clemino, M.; Dulieu, F.; Baumgärtel, H.; Leach, S. Photoion mass spectrometry of five amino acids in the 6-22 eV photon energy range. Chem. Phys. 2004, 298, 279-297

[57] Plekan, O.; Feyer, V.; Richter, R.; Coreno, M.; Prince, K.C. Valence photoionization and photofragmentation of aromatic amino acids, Mol. Phys. 2008, 106, 1143-1153

[58] Peptide Fragmentation Modeller, Biological MS Data and Software Distribution Center, Pacific Northwest National Laboratory

[59] Schlag, E.; W. Selzle, H. L.; Schanen, P.; Weinkauf, R.; Levine. R. D. Dissociation Kinetics of Peptide Ions, J. Phys. Chem. A 2006, 110, 8497-8500

[60] Horsch, P.; Urbasch, G.; Weitzel, K-M. Analysis of Chirality by Femtosecond Laser Ionization Mass Spectrometry. Chirality 2012, 24, 684-690

[61] Lux, C.; Wollenhaupt, M.; Bolze, T.; Liang, Q.; Köhler, J.; Sarpe, C.; Baumert, T. Circular Dichroism in the Photoelectron Angular Distributions of Camphor and Fenchone from Multiphoton Ionization with Femtosecond Laser Pulses. Angwe. Chem. Int. Ed. 2012, 51, 5001-5005 